\title{
Contraddizioni. II tempo delle città come deserti
}

\author{
Isotta Cortesi \\ DiArc, Università degli Studi di Napoli Federico II, Italia \\ isotta.cortesi@unina.it
}

\begin{abstract}
In questo tempo trascorso alla ricerca di soluzioni che ci consentano di conquistare nuovamente una condizione stabile per le nostre economie, per gli equilibri geopolitici della popolazione mondiale, siamo stati costretti ad acquisire maggiore coscienza del nostro presente, del fatto che la dimensione imperialista estrattiva nei confronti delle risorse del pianeta unita all'incremento della popolazione ha potenzialmente esaurito le opportunità di mobilità sociale e di effettivo progresso per gli individui e ancor più gravemente ha esaurito le risorse, nostra fonte di vita. Questo ci porta di fronte ad una grave condizione incontrastata di impoverimento, sociale, culturale ed economico. La spinta attuale verso una economia circolare che deve rapidamente soppiantare quella dei consumi in relazione alle pratiche virtuose, ma non prive di ombre, del Green New Deal ci offre, nel pieno dell'incerta evoluzione del COVID 19, l'opportunità di portare al centro della discussione la relazione di cura che gli individui devono instaurare con l'ambiente, con le risorse della natura e con il paesaggio, dal quale scaturisce una nuova alleanza biocentrica che ci consenta, grazie alla nostra capacità di adattamento, di 'resistere per rivivere'.
\end{abstract}

\section{Parole chiave}

Natura pubblica, etica ambientale, limitazioni, salute, contraddizioni, giustizia sociale, incertezza

\begin{abstract}
In this time spent searching for solutions that will allow us to once again attain a stable condition for our economies, for the geopolitical equilibrium of the world population, we have been forced to acquire greater awareness of our present. We have gained awareness on the extractive imperialist dimension, towards the planet's resources combined with population growth, has potentially exhausted the opportunities for social mobility and for effective progress for individuals. This has deeply exploited the natural resources, our source of life. This brings us to an undisputed condition of social, cultural and economic impoverishment. The current push towards a circular economy that must rapidly supplant that of consumption in relation to the virtuous, but not without shadows, practices of the Green New Deal offers us, in the midst of the uncertain evolution of COVID 19, the opportunity to bring the relationship of care. Individuals must establish a care attitude with the environment, with the resources of nature and with the landscape. A new biocentric alliance arises that allows us, thanks to our adaptability, to 'resist in order to relive'.
\end{abstract}

\section{Keywords}

Public Nature, Environmental Ethic, Limitations, Health, Contradictions,

Social Justice, Uncertainty

Received: December 2020 /Accepted: March 2021 | (c) 2020 Author(s). Open Access issue/article(s) edited by QULSO, distributed under the terms of the CC-BY-4.0 and published by Firenze University Press. Licence for metadata: CC01.0 
L'anno trascorso, con gli eventi ancora in atto, ha messo in evidenza, per tutti, quanto sia centrale e significativa la relazione tra l'uomo e la natura, quanto sia importante l'equilibrio tra gli esseri viventi e l'habitat che li accoglie e in particolare tra la salute dell'uomo e quella degli ecosistemi sui quali, attraverso il nostro fare, interveniamo.

Abbiamo in questo tempo recuperato una prospettiva evoluzionistica e dobbiamo allargare il nostro sguardo verso una visione del tempo profondo (Pievani, 2020, p.5). Quanto sta accadendo ha messo in luce il valore della relazione tra la salute dei viventi e il buon-essere, ossia il comportamento responsabile degli uomini, in relazione alla finitezza delle risorse, all'iniquità nella distribuzione delle stesse; e dei cittadini, in relazione alle strategie politiche di trasformazione delle città e dei paesaggi che per decenni hanno perseguito il saccheggio, ovverosia "la tragedia dei beni comuni" (Wilson, 2016, p.66).

Abbiamo compreso come sia importante discutere di salute della popolazione in stretta relazione alla salubrità dell'ambiente e dei suoi ecosistemi, alla loro accessibilità e anche alla qualità del progetto dello spazio aperto che si fa portatore dei valori dell'etica ambientale (Battaglia, 2002) poiché è indubbia la correlazione tra la diffusione dei virus dovuti alla zoonosi e la crisi ambientale globale.
In questo tempo sospeso inciso dalla paura del contagio, paura nutrita dai mezzi di comunicazione e disseminata con radicamenti diversamente profondi nella popolazione, abbiamo compreso che cosa implichi la privazione della libertà di relazioni e di movimento; abbiamo compreso gli effetti "dell'infodemia" (Floridi, 2020, p.268) sulla popolazione; abbiamo compreso cosa comporta la solitudine, la ripetitività delle giornate dentro e fuori la nostra casa, l'assenza delle relazioni stabili ed occasionali, la privazione del rapporto con lo spazio aperto nelle città e nei paesaggi. I nostri corpi hanno dovuto imparare i nuovi comportamenti nello spazio pubblico per la necessità di una distanza fisica tra noi e gli altri. I nostri corpi sofferenti di sedentarietà e isolamento, hanno subito certamente per questa stasi, un decadimento, un rallentamento, una sofferenza, insomma. I nostri visi sono spariti con le nostre espressioni e così abbiamo dovuto ricominciare a conoscerci.

I nostri centri storici, svuotati di persone, le strade, le piazze e i monumenti del nostro paese abitualmente assediate da visitatori desiderosi di immagini ed esperienze da riportare a casa, sono stati abbandonati e gli abitanti, prima increduli e poi affascinati, sono tornati ad avere con i loro paesaggi, anche quelli urbani, quella relazione di appartenenza e di cura che, forse, già era dei loro padri, ma della quale, in questo tempo recente erano stati privati. 
Così i corpi, spogliati di movimento e di esperienze, hanno cominciato a riconoscere e a ri-comprendere l'importanza della relazione con la natura e la necessità di svolgere una vita attiva nello spazio aperto per la propria salute e per il proprio benessere. Così oggi nei luoghi centrali del passeggio, ora deserti, appaiono uomini e donne che praticano sport correndo estraniati in attillati abbigliamenti tecnici, protetti dai suoni e dagli odori del mondo circostante privati di una vera esperienza, ma salvi dal traffico, fortunatamente molto ridotto, e mutati in corpi sfreccianti su monopattini, hoverboard, segway, bighe e ruote elettriche, operano ardite gimcane tra i pochi pedoni che animano lo spazio aperto. Questa diffusione di nuovi mezzi di trasporto per i corpi, negli spazi della città, è stata certamente favorita dalla riduzione del traffico in primis e dall'abbattimento delle polveri sottili nei centri urbani, dove l'inquinamento, nel periodo di confinamento forzato, si è ridotto cospicuamente. Ma anche nelle campagne periurbane le persone escono dalla città lungo le reti ecologiche rimaste integre e camminano per le strade sterrate dei campi, sugli argini e lungo i rii.

Questa pratica del 'ritorno alla natura' come esperienza si è consolidata durante questo anno di confinamento domestico, dove lo scorrere del tempo non è stato uguale per tutti, sia come necessità di momenti all'aria aperta per ritrovare una vita più sana, sia come acquisizione di responsabilità verso le risorse del pianeta, per un'etica ambientale.

Questa nuova relazione con la Natura pubblica esprime due temi: da una parte si riferisce alla necessità di considerarla come bene della comunità e, dall'altra rafforza il valore che le risorse della natura (suolo, acqua, vegetazione, fauna o altro) hanno acquisito nel nostro vivere quotidiano. Infatti, la Natura conforma le identità delle popolazioni e contribuisce al benessere fisico, psicologico della comunità. Parlare di Natura pubblica implica, da sempre, un ragionamento di responsabilità nella pratica del
La Natura pubblica è anche un assieme di ecosistemi abitato da molti e diversi esseri; essa contribuisce al benessere e alla salute non solo dei cittadini, ma di tutti i viventi e genera nuove forme e conoscenze che, nel progetto di architettura del paesaggio, interpretano i processi della natura (e non la imitano) per nutrire la dimensione culturale/educativa e compiere esperienze/conoscenze nello spazio aperto.

Oggi di fronte alla determinata fiducia riposta nel Green New Deal (Rifkin, 2019) è importante riconoscere che la sostenibilità del progetto non può essere limitata al mero soddisfacimento dei parametri quantitativi ecosistemici che per altro ci garantiscono la sopravvivenza, ma deve anche restituire centralità alla forma nell'esperienza del progetto in rapporto alla conoscenza (Meyer, 2008).

Nel nostro presente, dopo l'isolamento forzato di questo tempo sospeso, stiamo comprendendo il valore e la necessità del ripensare il progetto degli spazi aperti nelle città. Questo ci fa intuire come la Natura debba essere al centro della vita pubblica. La qualità del progetto nel nostro nuovo presente può divenire la congiunzione tra la dimensione estetica (oltre l'apparenza delle forme), la dimensione scientifica e l'etica ambientale. In questo modo si viene a nutrire quella visione altruistica, biocentrica e ambientalista che persegue le azioni necessarie volte a migliorare il futuro del mondo.

Le costrizioni imposte ai nostri stili di vita ci hanno offerto spunti per ripensare i ruoli e le forme degli spazi aperti della città, per investire sulla loro cura e sulla loro qualità. Ci chiediamo ancor di più oggi, alla luce degli accadimenti del 2020, come le esperienze di Natura possano migliorare i comportamenti negli esseri umani.

Le risorse sono certamente un servizio ecosistemico, un fattore quantitativo, funzionale a consentire il benessere e la salute dei cittadini poiché la quantità di vegetazione interviene direttamente sui fattori ambientali, riduce l'inquinamento e a incrementa la biodiversità. 
La questione ecosistemica e le discussioni attorno alla sostenibilità sono certamente fondamentali, ma rivelano, ancora una volta, quell'atteggiamento estrattivo' dove l'uomo si pone non in relazione paritetica, ma di superiorità alla natura che diviene immediatamente funzionale alle sue necessità. Questo habitus oggi non è più sufficiente poiché abbiamo compreso che è necessario istituire un dialogo tra pari, fondato sulle connessioni per spostare il campo dall'oggetto alla relazione. Siccome "pensare la natura è pensare un modello di società che la rende possibile, e viceversa" (Marrone, 2011, p.97) la dimensione relazionale ci fa comprendere chiaramente come la qualità dello spazio pubblico abbia effetti sulle strutture urbane, sulla comunità e sui comportamenti.

Possiamo forse ritenere che questo tempo sospeso ci abbia resi maggiormente consapevoli di come nei secoli nelle culture occidentali abbia prevalso sempre un atteggiamento estrattivo nei confronti delle risorse della natura, "il cui ridimensionamento era funzione del miglioramento della specie umana" (Marrone, 2011, p.95) e di come questo comportamento consolidato e condiviso abbia portato all'attuale profonda incertezza sul futuro degli uomini, specie capace di adattarsi ai cambiamenti che stiamo affrontando, ma che ha espresso, in questo recente apice, una profonda inadeguatezza. Se è vero che gli uomini "incapaci di ritrovare le capacità di giudizio e di rigenerare la creatività, le masse impoverite della società di mercato, ulteriormente sfiancate dalla pandemia [...] si scoprono indifese in un mondo senza punti di riferimento, un mondo in cui sono state lentamente sfibrate tutte quelle forze vitali, individuali e sociali, che contribuivano al loro benessere, o almeno a una vita degna" (Dentico, 2020, p.209) e allora diviene quanto più essenziale e necessario nutrire la dimensione biocentrica fondata sulla relazione paritetica degli esseri viventi, tutti.

Certamente il compito dei governi è garantire anche il benessere dei cittadini e fornire loro pari op- portunità per avere una vita attiva, quindi sana, nello spazio aperto. L'emergenza sanitaria mondiale sembra aver lasciato in secondo piano che "la Salute non -è- solo assenza di malattia, ma -è il-completo benessere fisico, mentale e sociale. La Salute è una risorsa della vita quotidiana"1. Purtroppo la qualità della vita quotidiana del nostro presente ha di fatto pregiudicato questo principio fondativo innescando effetti immediati sulla popolazione che non ha potuto e ancora stenta ad accedere ai servizi sanitari (non covid) con ripercussioni estremamente gravi sulla salute, che ha visto una vera e propria rivoluzione antropologica nelle relazioni, deprivata di alcune abitudini ed usi ne ha escogitate subito altre come necessario adattamento ed ha riscoperto il piacere della lentezza, del camminare e del trascorrere il tempo all'aperto fuori, insieme, anche nello spazio di Natura.

Queste considerazioni sono alla base della rete delle Biophilic Cities ${ }^{2}$, esse condividono la fiducia nella relazione biocentrica tra gli spazi urbani, gli abitanti e i sistemi naturali (fiumi, laghi, montagne...) che hanno dato origine agli insediamenti; Tim Beatley riferisce che "ogni città ha una stretta relazione con la Natura, ogni città è in sé biofila. Ogni città ha la possibilità di incrementare questa condizione, questo richiederà cambiamenti intenzionali nelle politiche, nelle pratiche urbanistiche e nelle visioni di strategia urbana che saranno volte ad incrementare la qualità di Natura nelle città del XXI secolo".

La questione centrale non è veramente la necessità di cambiare i nostri stili di vita, ma è piuttosto come trarre ispirazione dai valori percepiti durante quest'anno di transizione, per avviare il nostro necessario adattamento. Certamente oggi abbiamo bisogno non solo di ripiantare alberi ovunque per raggiungere quei benefici climatici che rigenerano la biodiversità, ma soprattutto dobbiamo ripensare a come re-inserire, nel cerchio della vita, l'uomo per riscoprire le virtù della benevolenza verso qualsiasi forma di vita (Cabanes, 2017, p.27). 
Apparentemente queste affermazioni possono sembrare ingenue, ma esprimono la stringente necessità di unire politiche ambientaliste verdi (la green economy e la share economy) con politiche digitali blu a supporto di un'economia dell'esperienza e non più del consumo, radicata sulla qualità delle relazioni e dei processi e non sulle cose e la loro proprietà, per sostenere l'ambiente e costruire una società migliore (Floridi, 2020, p.8, p.12) in una visione traslazionale, ossia capace di tenere insieme i saperi, dalla dimensione teorica a quella pratica, legati da una continuità determinata dall'interesse per una positiva risoluzione dei problemi. In questa discussione diviene quindi fondamentale perseguire la qualità del progetto dello spazio aperto che abitiamo, inteso come lo spazio delle relazioni in un circolo virtuoso tra natura e umanità. Infatti questo è il tempo di un 'ecologismo umanista e scientifico'. "Umanista perché gli interessi della natura e della specie umana coincidono completamente. Scientifico perché basato su evidenze" (Pievani, 2020, p.6) che registrano i cambiamenti in atto.

Oggi consideriamo "il prendersi cura" come quell'azione principale e indispensabile che stiamo mettendo in atto a scala nazionale. Questo processo, del prendersi cura, accade anche in quei progetti di paesaggio che generano e risanano gli ecosistemi mentre migliorano la relazione tra l'uomo e lo spazio che abita.

La Natura ha un'endogena capacità operante, ossia mette in atto l'azione riparatrice di cura sull'ambiente e sulle persone, risana i processi ecologici e tramite la sua bellezza incrementa il benessere psico-fisico della popolazione. "Coloro che vivono tra le bellezze e i misteri della terra -siano essi scienziati o persone comuni- non saranno mai soli o stanchi della vita. C'è qualcosa di infinitamente terapeutico nel continuo ripetersi della natura" (Carson, 2020, p.33) Come conseguenza alla preclusione della vita attiva dovuta ai condizionamenti imposti dal virus, la co- munità degli uomini e donne ha rivalutato, dopo il confinamento, il valore della relazione con la natura trascorrendo molto tempo all'aperto.

In particolare, come architetti del paesaggio affermiamo la rilevanza del progetto nell'osservare esempi straordinari che hanno rigenerato sia gli spazi aperti, luoghi di nuove esperienze per gli individui e, allo stesso tempo, hanno vivificato gli ecosistemi come la rinaturalizzazione del fiume Aire (Descombes \& Rampini, 2016) che riporta l'alveo alla sua conformazione originale e utilizza il canale come riferimento per documentare e trasmettere l'evoluzione del paesaggio; o il progetto di adattamento climatico del parco Hans Tavsens e Korsgade a Inner Nørrebro (SLA Copenhagen, 2016) che esprime la riconciliazione tra i cicli del paesaggio e quelli degli uomini come avviene anche nel parco Luming a Quzhou (Turenscape, 2015) e nel recupero dell'ecosistema del fiume Sanlihe (Turenscape, 2010); mentre nel Parco delle acque di Saragoza, si struttura un nuovo ecosistema igrofilo capace di intercettare le acque del fiume Ebro per depurarle e renderle balneabili (C. Dalnoky, 2008), ma anche il Waller Creek (M. Van Valkenburgh Associates, 2019) che tramite il progetto, incrementa la comprensione per la popolazione della relazione con la natura, attiva e operante. Ma certamente il paradigma progettuale (capace di affermarsi sia per la qualità della natura progettata sia per la vita dell'uomo) consolidato nella storia del paesaggio contemporaneo è l'Allegheny Riverfront (M. Van Valkenburgh Associates, 1998) dove la conquista dello spazio pubblico risolve i dislivelli con percorsi per l'uomo e progetta la fascia di contatto con il fiume per ricomporre gli ecosistemi ripariali. Certamente la ricerca espressa in Living Break Waters (K. Orff_Scape, 2014) esprime la centralità del processo traslazionale tra ecosistemi, individui, sistemi produttivi e risanamento ambientale in un processo che rigenera i luoghi, le catene alimentari e gli ecosistemi per la costruzione di un'ecologia urba- 
na (K. Orff_Scape, 2016) dalla quale ripartire per considerare la città come un organismo vivente.

I progetti elencati sono vocati a esprimere in modo esemplare i temi della discussione poiché intervengono su diversi fronti per incrementare i parametri ambientali, ma non si accontentano di essere una dotazione funzionale e quantitativa (semplicemente come spazi contenitore di attività o espressione di un progetto-programma): essi vanno al di là del parametro scientifico per assurgere a riferimento culturale, che mentre contribuisce al benessere dei cittadini e dell'ambiente, amplifica la conoscenza in relazione alla cura dei luoghi e alla vocazione comunicativa del progetto.

Coloro che trasformano gli spazi abitati (e tra questi annoveriamo i politici, gli urbanisti, le associazioni di cittadini, gli architetti e i paesaggisti...) hanno acquisito oggi, grazie agli eventi legati a questa pandemia, quella maggiore consapevolezza sulle responsabilità che l'uomo ha in relazione al futuro, alle incertezze del presente e alla possibilità di innescare processi virtuosi di rivoluzione tra Natura pubblica e informazioni digitali per migliorare sia la salute della popolazione sia le condizioni delle risorse del pianeta e interrompere quell'atteggiamento estrattivo e predatorio che ha contraddistinto l'uomo in questi ultimi secoli per innescare invece comportamenti virtuosi di cura e d'integrazione, volti a ricomporre in modo traslazionale le relazioni tra i viventi. Oggi è molto importante rafforzare la riconoscibilità (per significato e forma) del progetto (promuovendo così stili di vita più salutari), mentre devono rafforzarsi le responsabilità e le competenze dei progettisti in relazione all'utilizzo delle risorse finite del patrimonio della Natura.

Quasi senza indugi si può affermare che il presente ha la necessità di mettere in atto la pratica della cura nelle esperienze degli individui e delle comunità. Questo approccio privilegia una visione di responsabilità a lungo termine per il futuro piuttosto del perseguimento di una gratificazione immediata e estrattiva volta al consumo irresponsabile delle risorse (Way, 2016, p.138) che ha principalmente caratterizzato il nostro recente passato.

La difficoltà ancora non completamente superata ci ha fatto comprendere come sia importante per il presente e per il nostro futuro, la cura dei luoghi attraverso la relazione uomo-insediamento-natura e come il progetto di paesaggio, nella sua intrinseca vocazione riparatrice, abbia dirette implicazioni per la salute e il benessere degli ecosistemi e degli abitanti. Secondo questa visione si rafforzano le relazioni tra lo spazio urbano e il paesaggio, gli ecosistemi e gli abitanti. Questo processo traslazionale attraverso i saperi genera la cultura della responsabilità che con processi virtuosi ha effetti diretti ed efficaci sulla salute e sul benessere dei cittadini e degli ecosistemi che consentono la vita. 


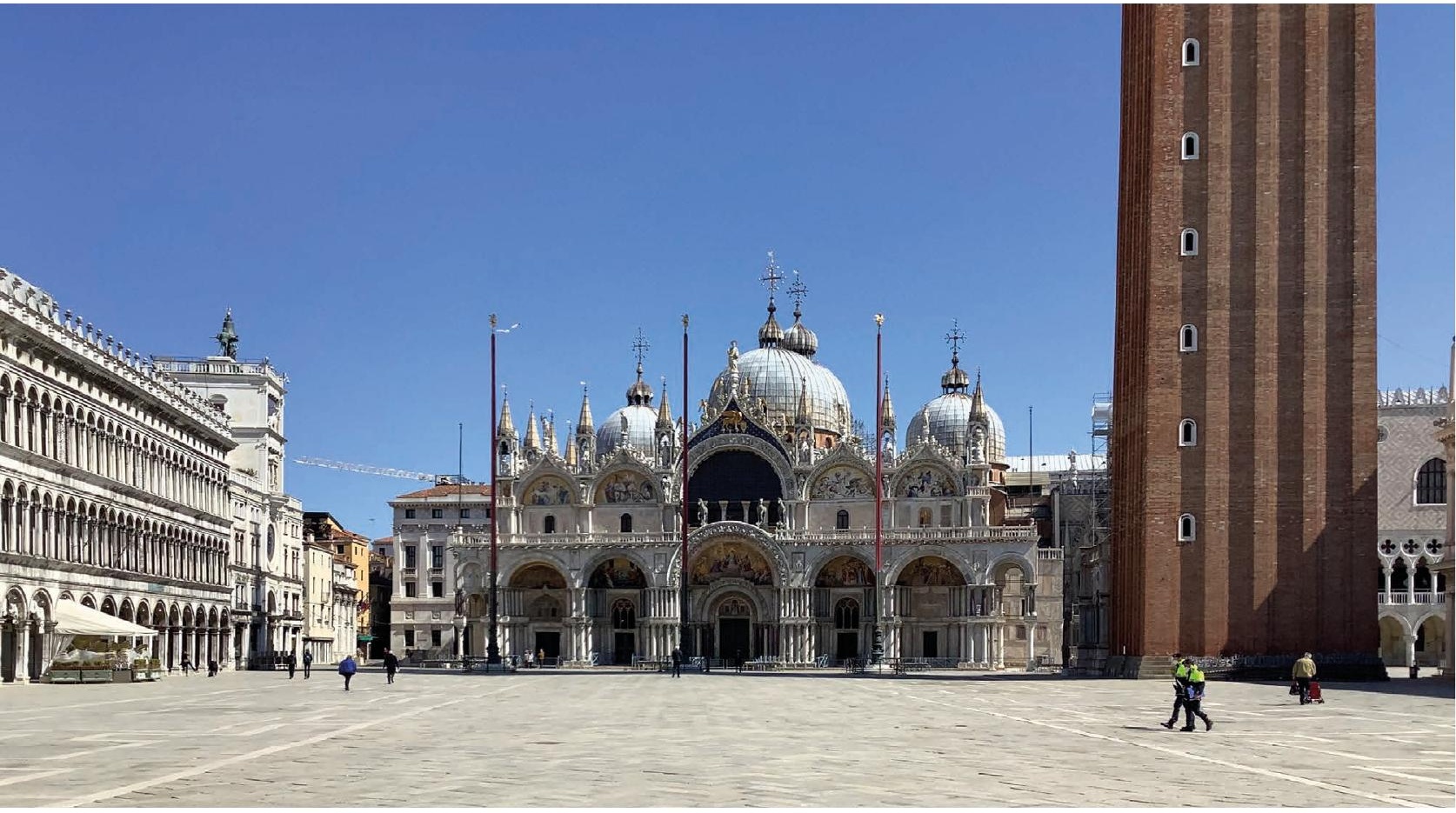

Fig. 1 - Venezia, Piazza S. Marco, Domenica di Pasqua 2021, ore 12.52. Campo.
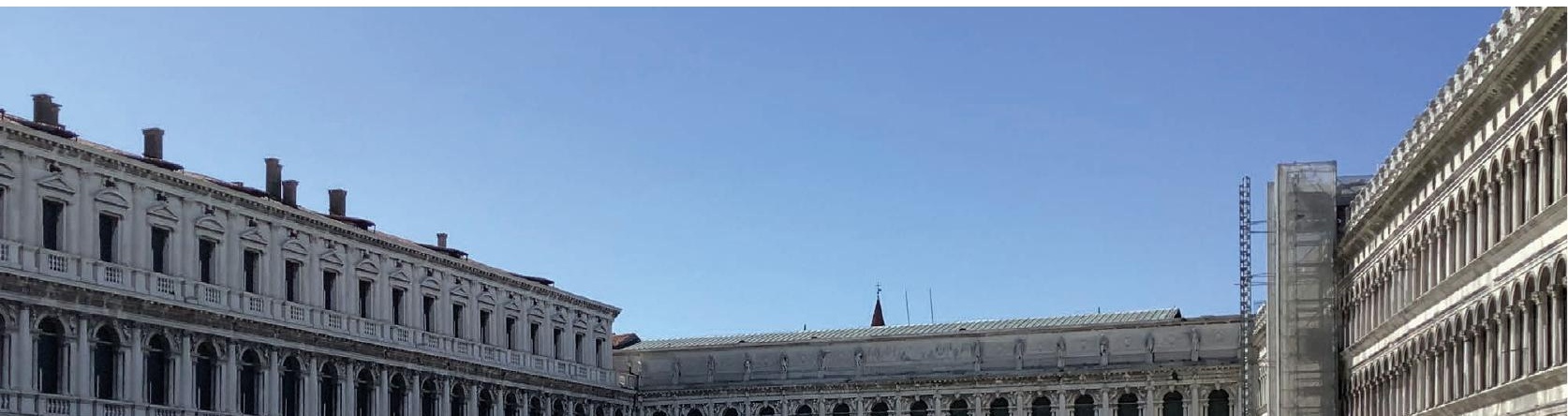

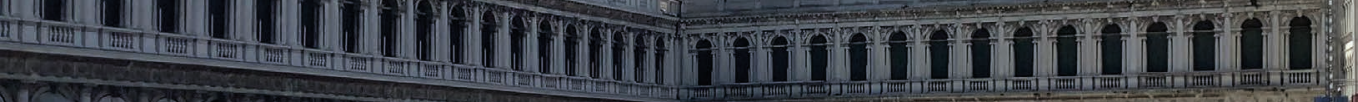

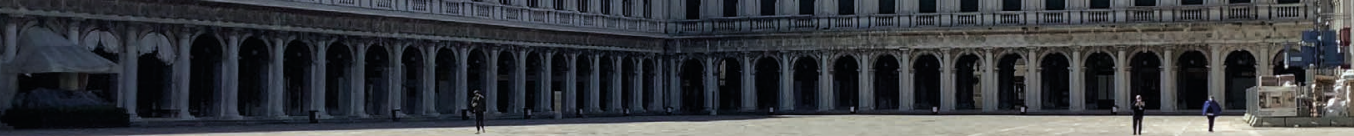

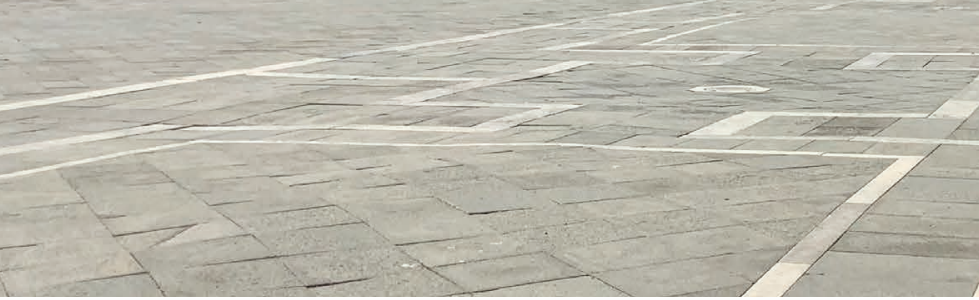

Fig. 2 -Venezia, Piazza S. Marco, Domenica di Pasqua 2021, ore 12.54. Contro-Campo. 


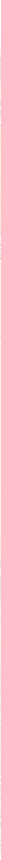

Fig. 3 - Venezia, Canal Grande, Lunedi di Pasqua 2021, ore 17.35. Silenzio.

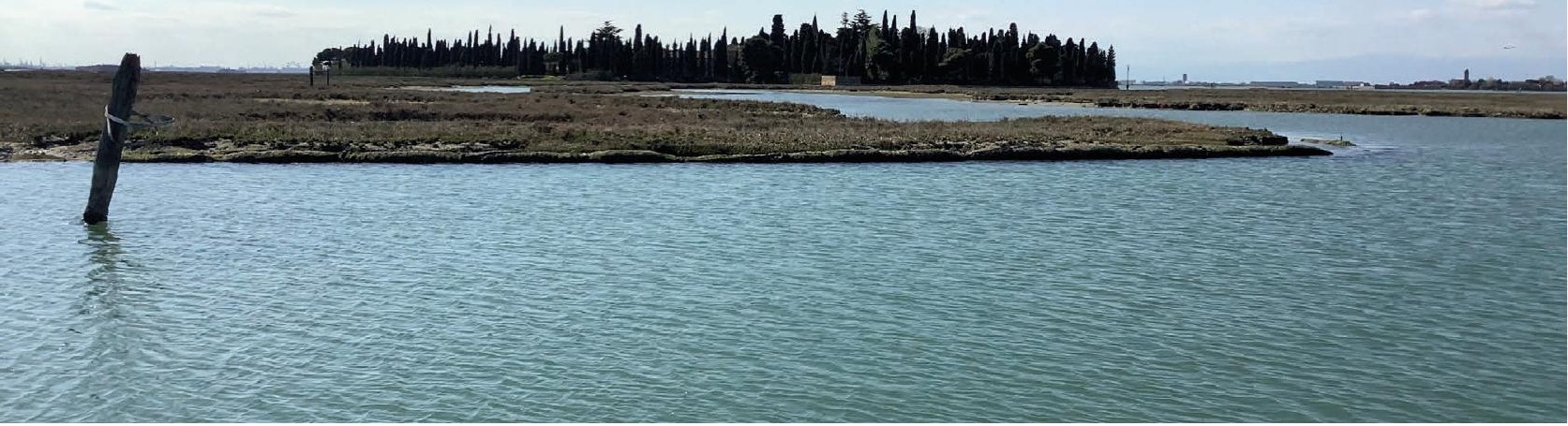

Fig. 4 -Venezia, S. Francesco del Deserto, Pasqua 2021, ore 16.14. Barene e frati eremiti nella laguna. 


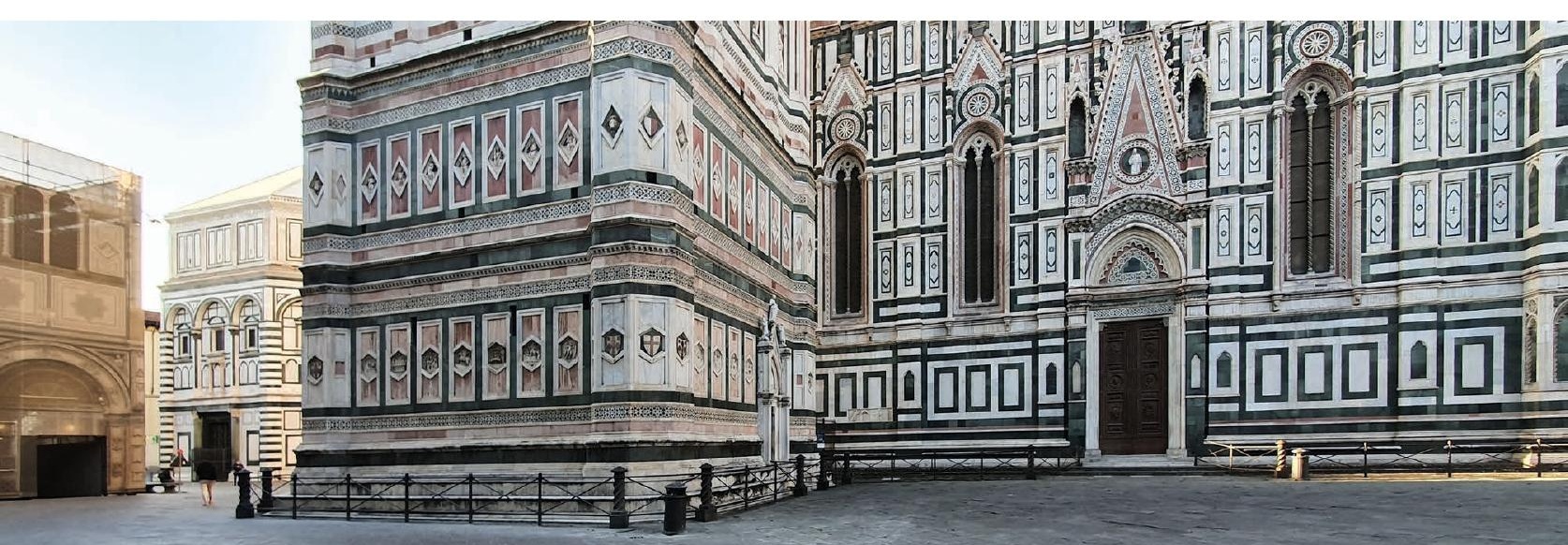

Fig. 5 -Firenze, Piazza del Duomo, 9 Aprile 2021, ore 18.49

Battistero di S. Giovanni, paramenti murari della Cattedrale e del Campanile di S. Maria del Fiore.

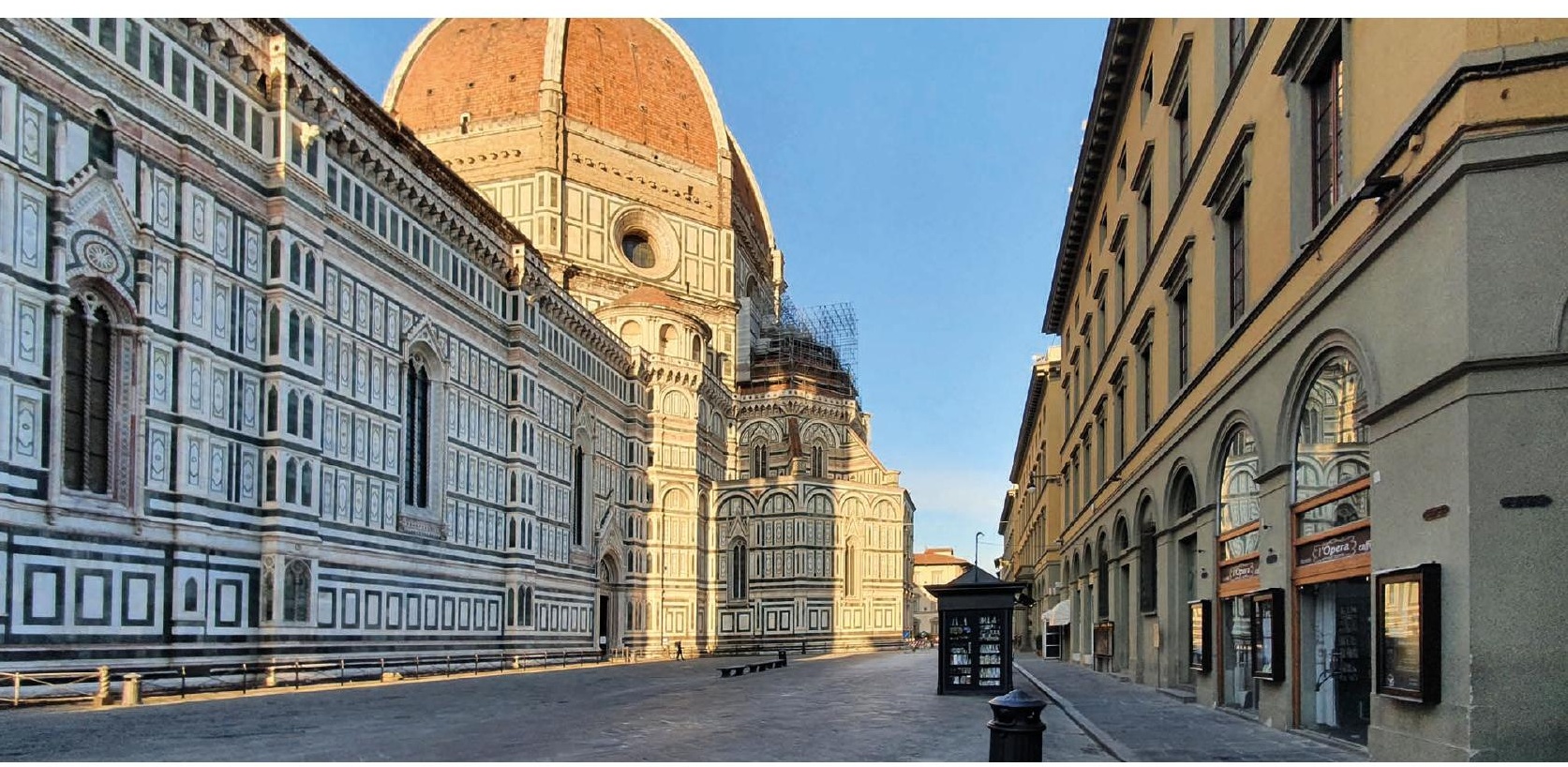

Fig. 6 -Firenze, Piazza del Duomo, 9 Aprile 2021, ore 18.54. II passeggio. 


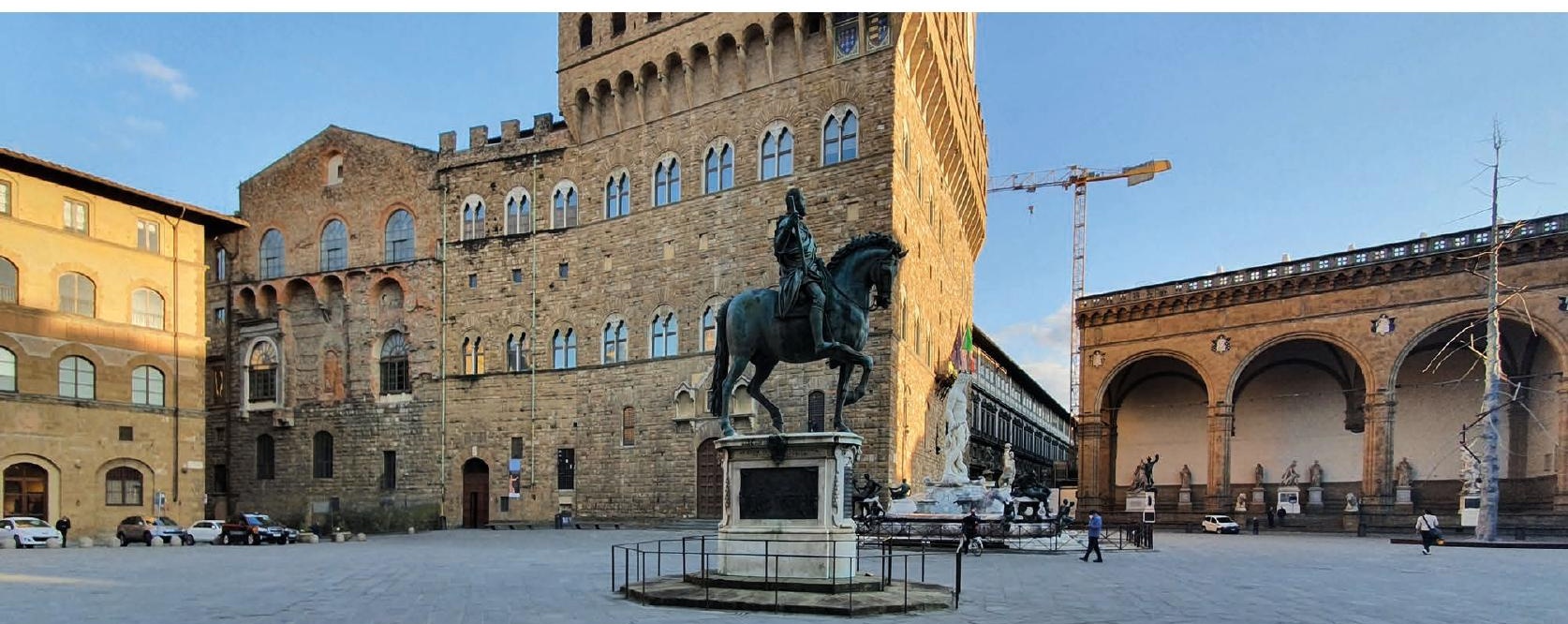

Fig. 7 -Firenze, Piazza della Signoria, 9 Aprile 2021, ore 19.04, Cosimo I, "Abete" (Spelacchio) e la gru.

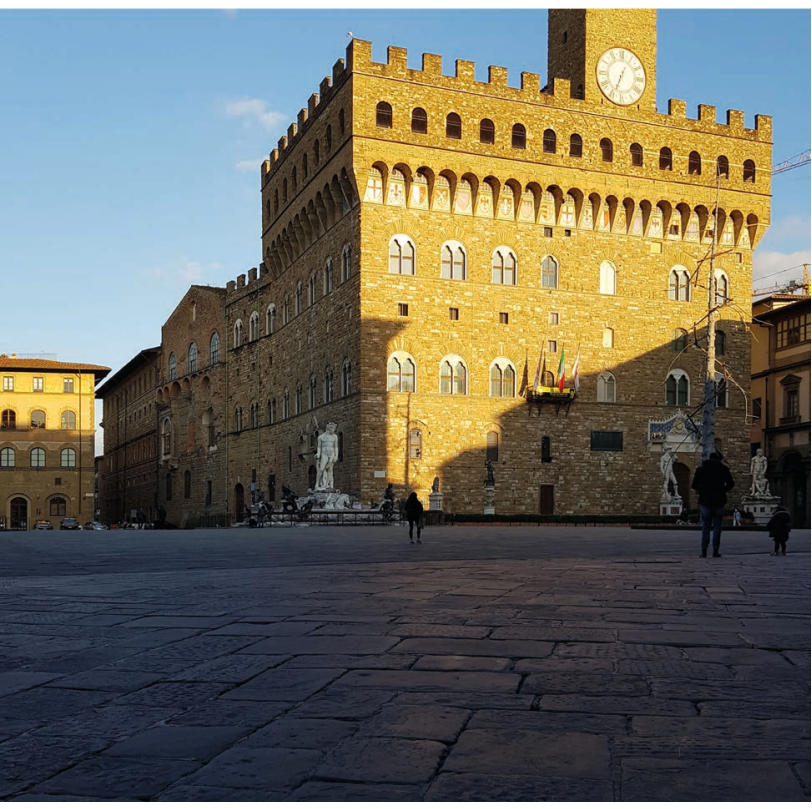

Fig. 8 - Firenze, Piazza della Signoria, 9 Aprile 2021, ore 19.01 Ombre, Nettuno e Palazzo Vecchio.

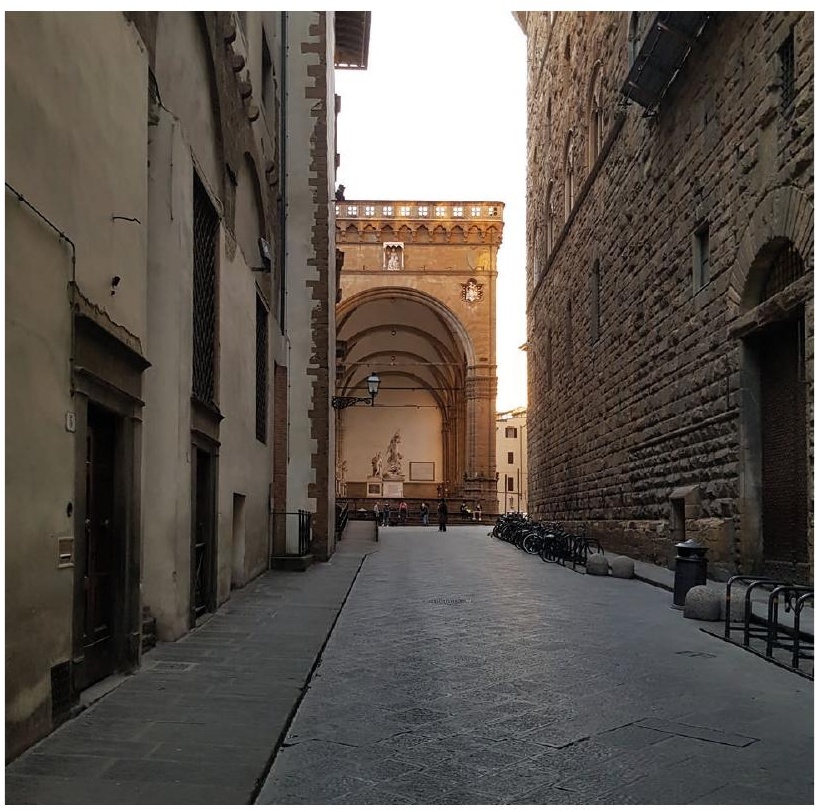

Fig. 9 - Firenze, via della Ninna, 9 Aprile 2021,

ore 19.07, II Ratto di Polissenna, la Loggia e il Palazzo. 


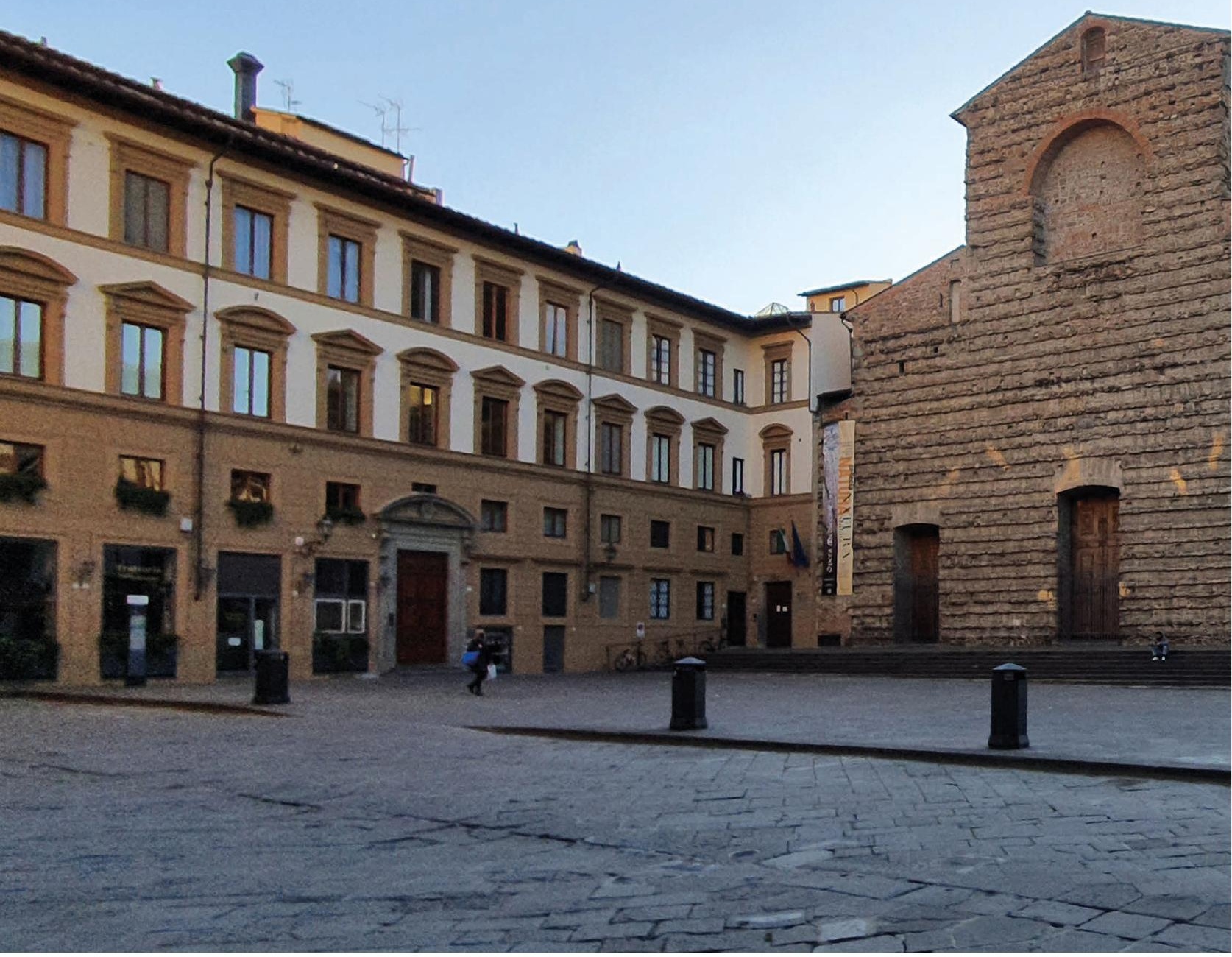

Fig. 10 - Firenze, Piazza S. Lorenzo, 9 Aprile 2021, ore 18.48,

Giovanni dalle Bande Nere, Filippo Brunelleschi e Michelangelo a S. Lorenzo.

pagina a fronte

Fig. 11 - Firenze, via dell'Ariento, 9 Aprile 2021, ore 18.54,

Il Mercato di S. Lorenzo. 

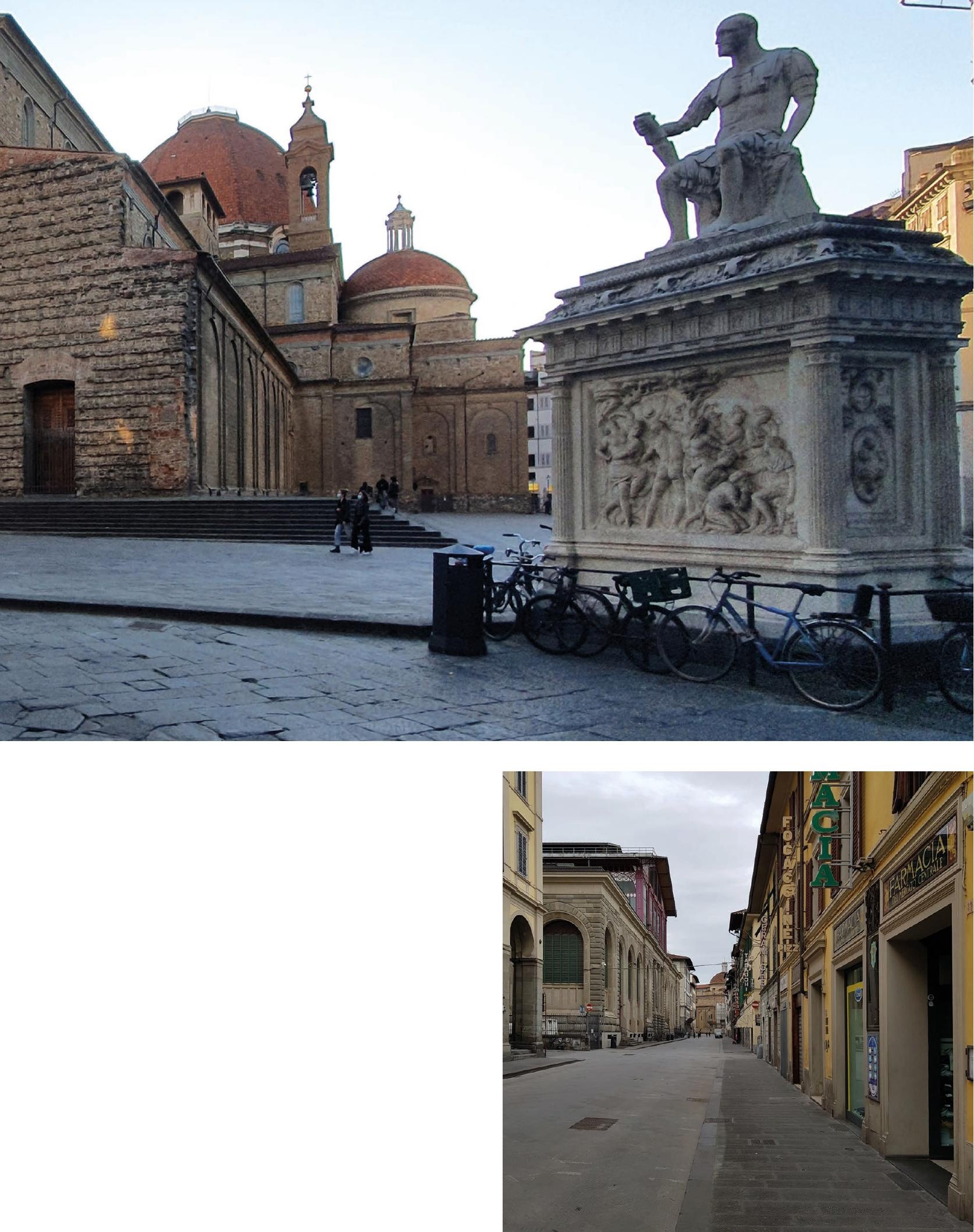


\section{Note}

1 'Carta di Ottawa per la promozione della Salute' - I Conferenza Internazionale sulla promozione della Salute, 1986.

${ }^{2}$ The Biophilic Cities Project si riferisce al lavoro di ricerca internazionale sulle città biofile, coordinato dal professor Tim Beatley e dal suo team alla School of Architecture dell'University of Virginia. 


\section{Bibliografia}

Battaglia L. 2002, Alle origini dell'etica ambientale. Uomo, natura, animali in Voltaire, Michelet, Thoreau, Gandhi, Dedalo, Bari.

Cabanes V. 2017, Homo natura, En harmonie avec le vivant, Libella, Parigi.

Carson R. 2020, Brevi lezioni di meraviglia, Aboca, San Sepolcro, (The Sense of Wonder, prima edizione 1956).

Cortesi I. 2020, Natura pubblica in 40 parole per la cura della città. Lessico dei paesaggi della salute, a cura di Criconia A., Cortesi I., Giovannelli A., Quodlibet, Macerata.

Dentico N. 2020, Ricchi e buoni? Le trame oscure del filantrocapitalismo, Emi editore, Verona.

Floridi L. 2020, II verde e il blu. Idee ingenue per migliorare la politica, Raffaello Cortina Editore, Milano.

Franzen J. 2020, E se smettessimo di fingere?, Einaudi, Torino.

Liberti S. 2020, Terra bruciata. Come la crisi ambientale sta cambiando l'Italia e la nostra vita, Rizzoli/Mondadori, Milano.

Marrone G. 2011, Addio alla Natura, Einaudi Editore, Torino. Morandini S. 2009, 'Natura in etica ambientale', in Botturi F. Mordacci R. (a cura di) 2009, Natura in etica, Vita e Pensiero, Milano, pp.191-211.

Meyer E. K. 2008, Sustaining beauty. The performance of appearance. A manifesto in three parts, «Journal of Landscape Architecture», Volume 3, Issue 1.

Orff K. - SCAPE 2016, Toward an Urban Ecology, The Monacelli Press, New York.

Pievani T. 2020, Planet Book, Roberto Koch editore, Roma.

Rifkin J. 2019, Un Green New Deal Globale. II crollo della civiltà dei combustibili fossili entro il 2028 e l'audace piano economico per salvare la terra, Mondadori, Milano.
Way T. 2016, Urban Ecological Design as Feminist Practice, in Orff K.-SCAPE 2016, Toward an Urban Ecology, The Monacelli Press, New York, pp.132-138.

Wilson E.0. 2016, Metà della terra. Salvare il futuro della vita, Codice edizioni, Torino. 